$S \angle A C-T N-96-1$

SLAC-TN-96-1

March 1996

\title{
Documentation for vlasov, version 0.1 *
}

\author{
J. Scott Berg \\ Stanford Linear Accelerator Center, Stanford University, Stanford, CA 94309
}

\begin{abstract}
This paper describes how to use the code vlasov, which implements the computation of multibunch frequencies and growth rates when the effects of multibunch mode coupling are included. The theory behind this code is described in [BR95a, BR95b, Ber96]. This paper also describes the algorithm used to compute the modes, and the approximations made.
\end{abstract}

\section{Running the Code}

The program modes 00 will create a binary file output file from the input file described in section 2 . To view this output, use the program ploto0.

\section{1 modes 00}

The syntax for running modes 00 is simply

modes00 file.in file.out

where file.in is the input file described in section 2, and file. out is a binary file containing the computed modes. Note that the program will exit if file.out already exists.

\section{2 plot00}

To run plot00 to make a plot of the modes, type

plot00 file. out file.xmgr

where file.out is the output file generated from modes00, and file.xmgr is a file which can be given to the program xmgr to produce a plot of the modes. Note that the program will exit if file.xmgr already exists. To load the plot into xmgr, say

xmgr file.xmgr

When running plot00, you will be prompted for the type of plot that you want. The opening menu will look like:

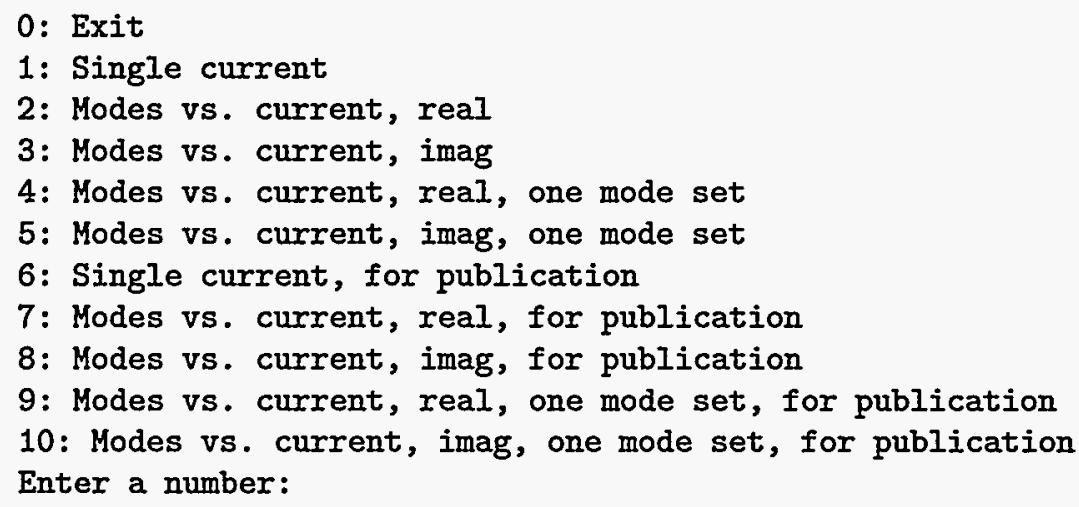

-Work supported by Department of Energy contract DE-AC03-76SF00515. 


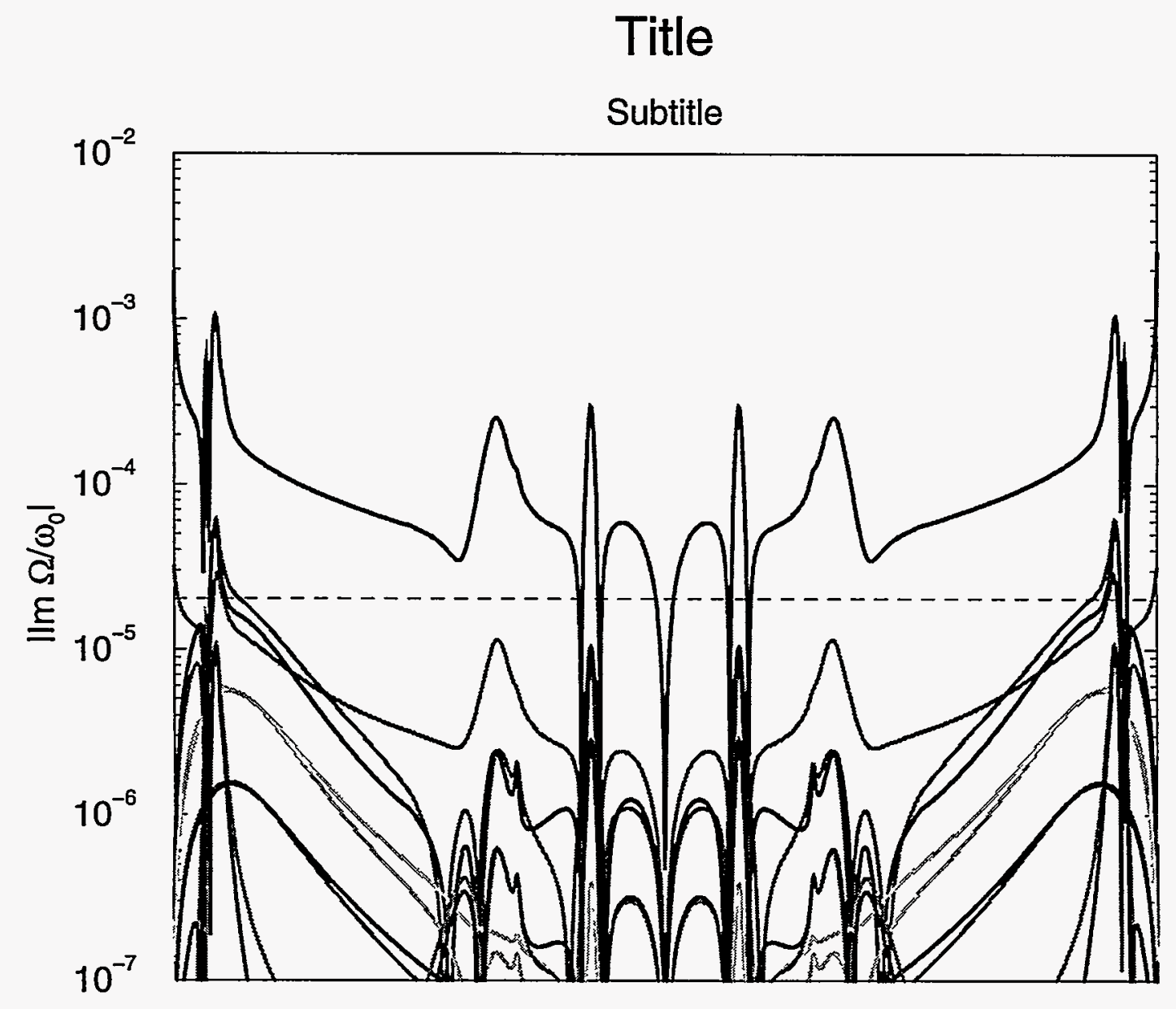

Multibunch Mode Number

Figure 1: Output from "Single current" and "Absolute value of imaginary part."

In general, options 1, 2, and 5 are the most useful. The corresponding "for publication" options are attempts to produce a plot that will satisfy Physical Review.

If you select option 1, you will be prompted for another choice:

1. Absolute value of imaginary part

2. Absolute value of shift

3. Positive imaginary part

Enter choice:

Option 1 will give you a plot similar to figure 1 . Option 2 will give you a plot similar to figure 2 . This is useful for the estimating of Landau damping thresholds. Option 3 will give you a plot similar to figure 3 . In all cases you are prompted for a damping rate, which will give a dashed line on the resulting plots.

If you selected option 2 from the main menu, "Modes vs. current, real," then a plot will be produced similar to figure 4. The plot shows the real parts of the frequencies for all the modes. If you selected option 3, "Modes vs. current, imag," then a plot will be produced similar to figure 5 . This shows the imaginary parts of the frequencies for all the modes. As you can see, this plot is often not particularly useful, except in the case of a single bunch $(M=1)$. It is generally more useful to plot the imaginary parts of specific modes. This is done through option 5, "Modes vs. current, imag, one mode set." In addition to the 


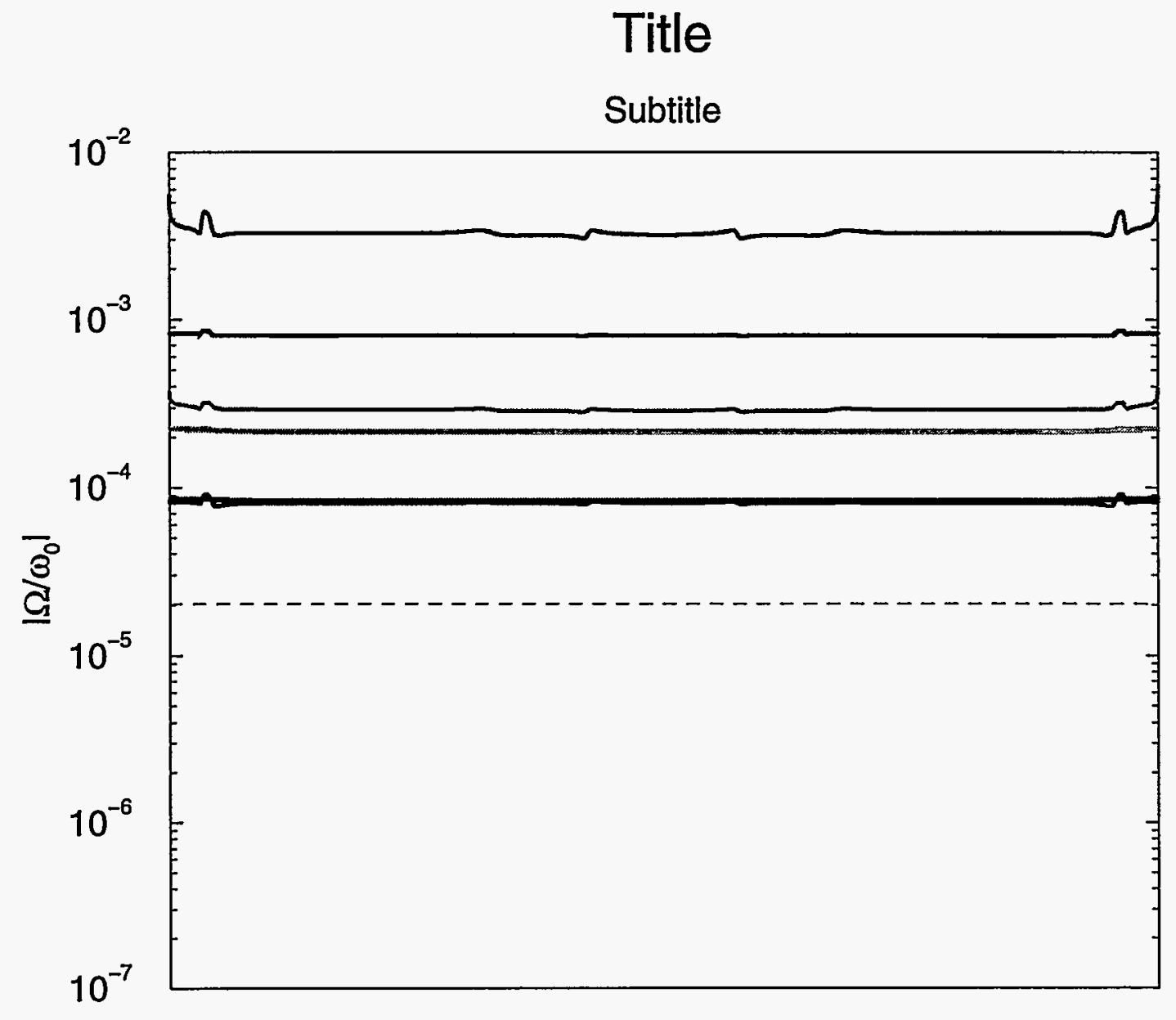

\section{Multibunch Mode Number}

Figure 2: Output from "Single current" and "Absolute value of shift."

usual prompts, you will also be prompted for a mode number. The current method of numbering these is a bit cryptic. The mode number you are prompted for should be the value of $\left(n+n^{\prime}\right) / 2$ for that mode (these values are described in [Ber96]; see equation (4.79) ). For a non-radial mode, if the zero-current frequency is $\omega_{y} \pm m \omega_{s}$, than $m$ is the number you specify. However, for a radial mode, the number you specify is $m+2 k$, where $k$ is the order of the radial mode. For instance, for the first $m=1$ radial mode, you would specify 3 . If necessary, the program will then prompt you for the azimuthal offset. This is always the value of $m$. A plot will be produced that is similar to figure 6 .

\section{Input Format}

The input file for the program consists of several keywords followed by values. The keywords must be typed exactly: case does matter! The order of the keywords is irrelevant. The keywords may be followed by one or more values, depending on the keyword. Those values may appear anywhere after the keyword, even on the next line. They are separated from one another by any combination of spaces, tabs, and end-of-line characters. 


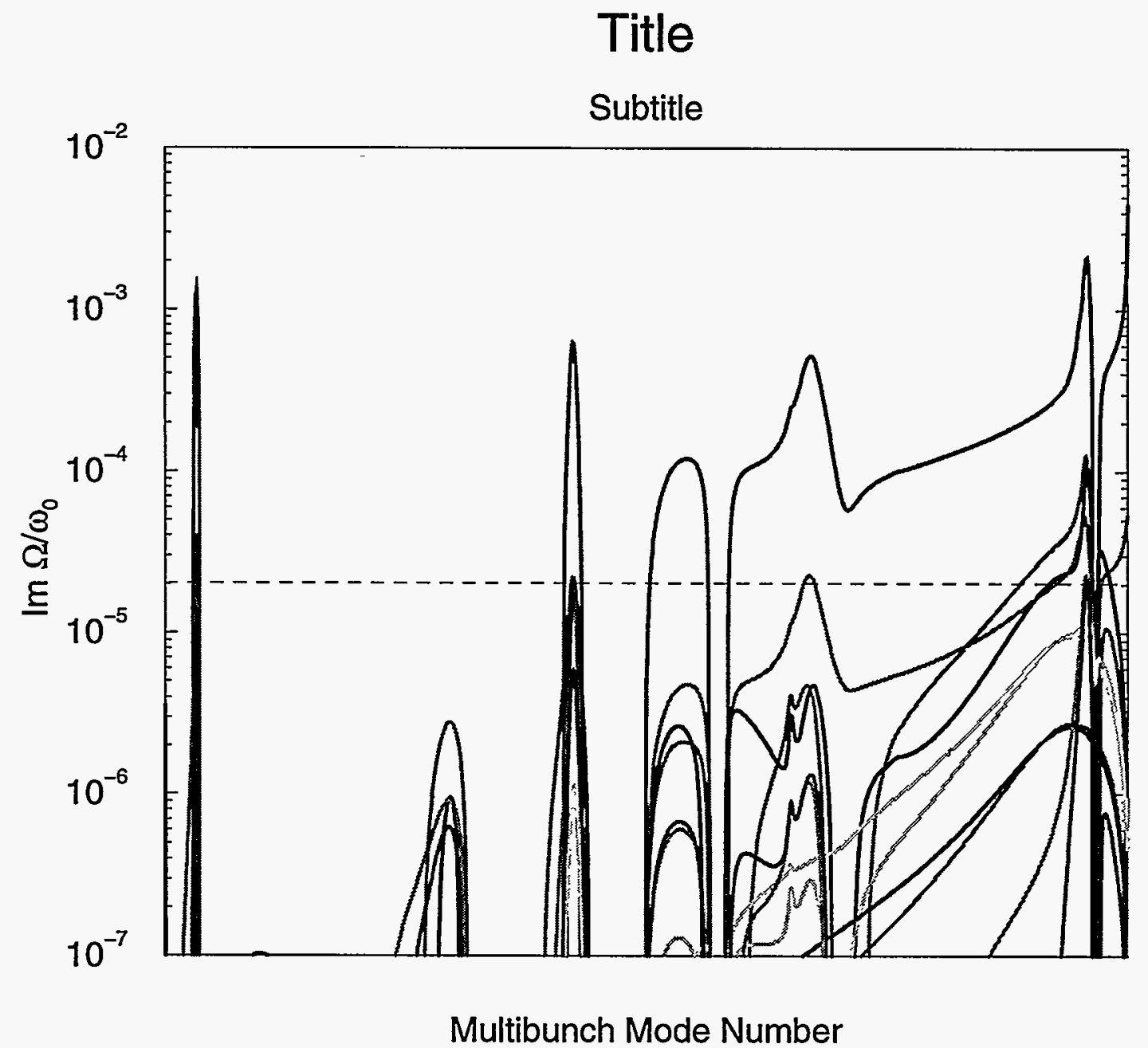

Figure 3: Output from "Single current" and "Positive imaginary part-."

\subsection{Parameters}

All the values described in this section are required, unless otherwise indicated. Just to be clear, the keywords do not contain periods; that's just my punctuation in this paper.

Momentum_Compaction. This is followed by a single number. This is the negative of $\eta_{c}$ as defined in [Wie93]. It should be positive above transition. The program will also accept "eta_c." This part is optional.

Betatron_Tune. This is followed by a single number. The program will also accept "nu_beta."

Synchrotron_Tune. This is followed by a single number. The program will also accept "nu_s."

Bunch Length. This is followed by a single number. It is the bunch length divided by the circumference of the ring. The program will also accept "sigma. $1 . "$

Energy. This is followed by a single number. It is the energy in eV. The program will also accept "E_0."

Current. This is followed by two numbers: the maximum current, and the number of current points to plot. The program will also accept "I." 


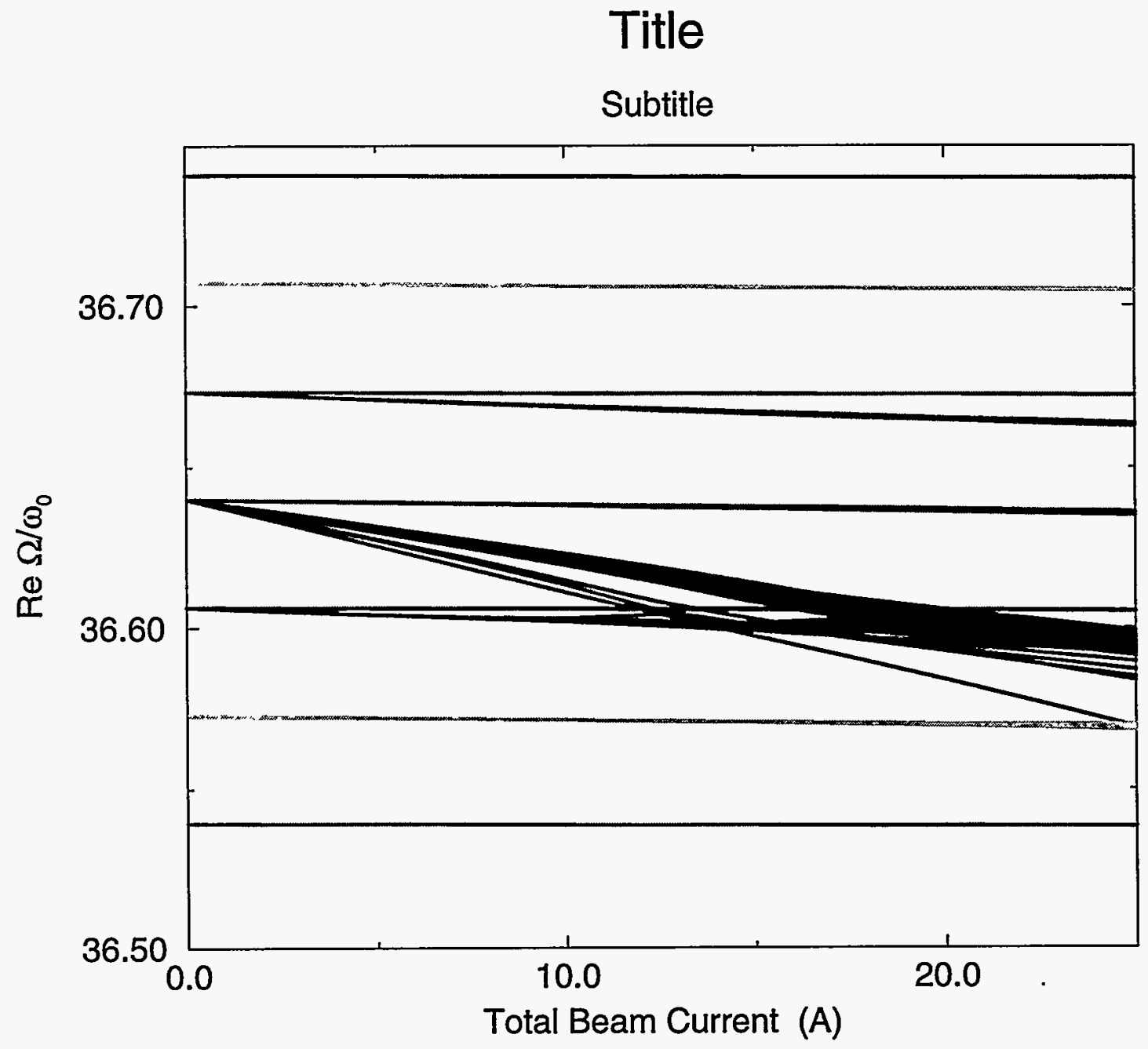

Figure 4: Output from "Modes vs. current, real."

Emittance. This followed by a single number: the transverse emittance of the beam divided by the circumference of the ring. Emittance is defined such that $\sigma_{y}=\sqrt{\epsilon_{y} \beta_{y}}$. The program will also accept "epsilon." This part is optional.

n. This is followed by two positive integers: the minimum and maximum azimuthal mode numbers. Note that the radial modes which have the same form factors as some non-radial modes are always computed together with those modes. So, for instance, including an azimuthal mode number of 3 always include the $m=1$ radial modes as well. Currently, the program won't work unless the minimum mode number is 0 .

p. This is followed by three integers: the minimum mode index offset, the step in that offset, and the total number of offsets which will be computed.

M. This is followed by one positive integer, the number of symmetric bunches to do the computation for. Currently the program only works with symmetric bunches.

Chromaticity. This is followed by one number, the chromaticity. This part is optional.

Transverse. What follows this is a description of the transverse impedance. This will be described in a separate section. This part is ended by the word "END." This part is optional. 


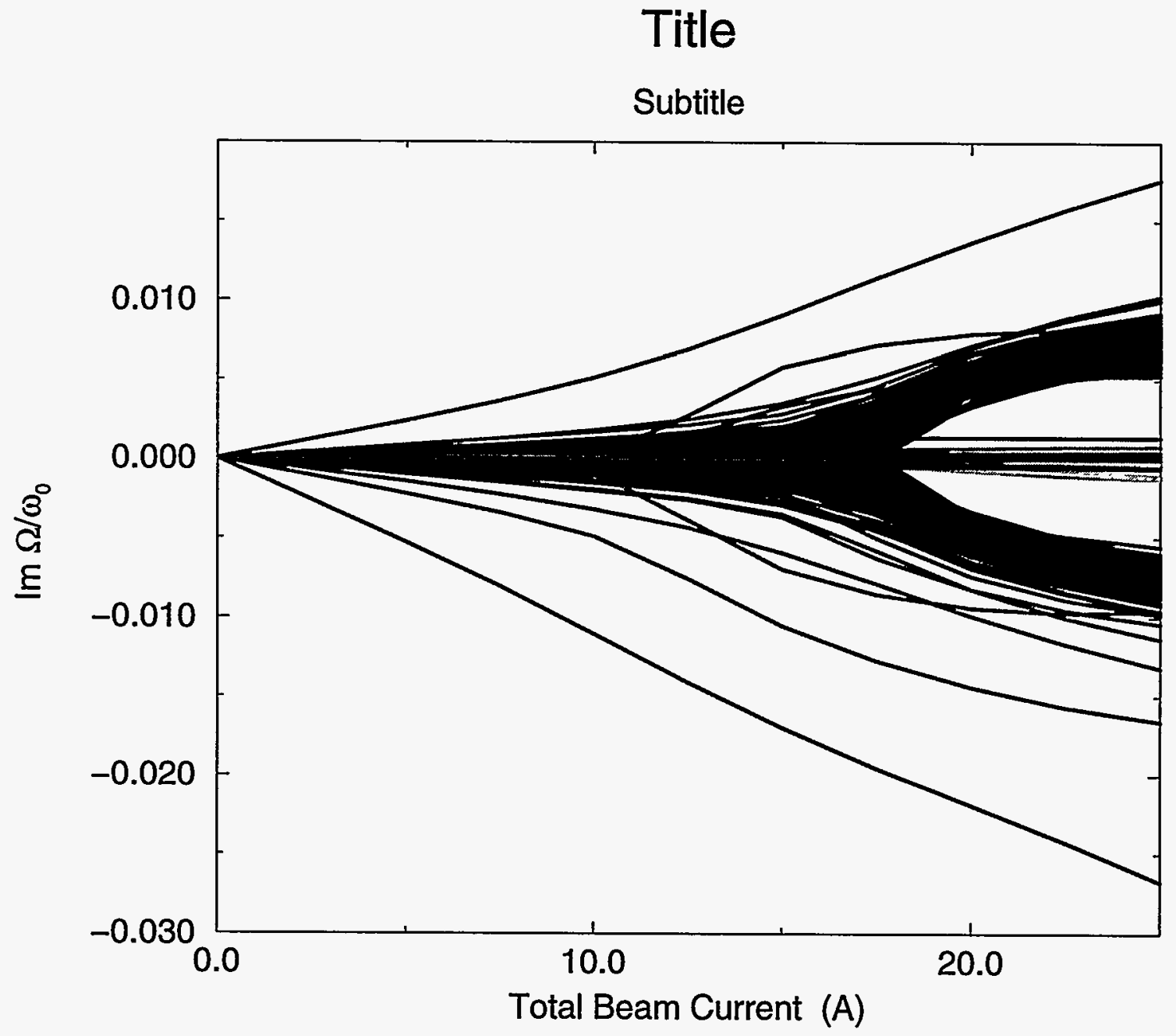

Figure 5: Output from "Modes vs. current, imag."

Transverse.Feedback. What follows this is a description of the transverse feedback system. This will be described in a separate section. This part is ended by the word "END." This part is optional.

\subsection{Transverse Impedance}

Between the Transverse and END keywords, there are a sequence of keywords which indicate what type of transverse impedance you are using. Each of these keywords will be followed by one or more values. One of these values will be the strength of the impedance. This value will be in units of Ohms. This value is the actual impedance multiplied by the average transverse $\beta$-function over the impedance. The user must compute this product and use it in the appropriate place. Finally, all frequencies used are the actual frequency divided by the revolution frequency.

Resonator.II. There are three values, $\omega_{R}, Q$, and $R$, where the impedance is given by

$$
\frac{R}{\omega / \omega_{R}+i Q\left(1-\omega^{2} / \omega_{R}^{2}\right)} \text {. }
$$




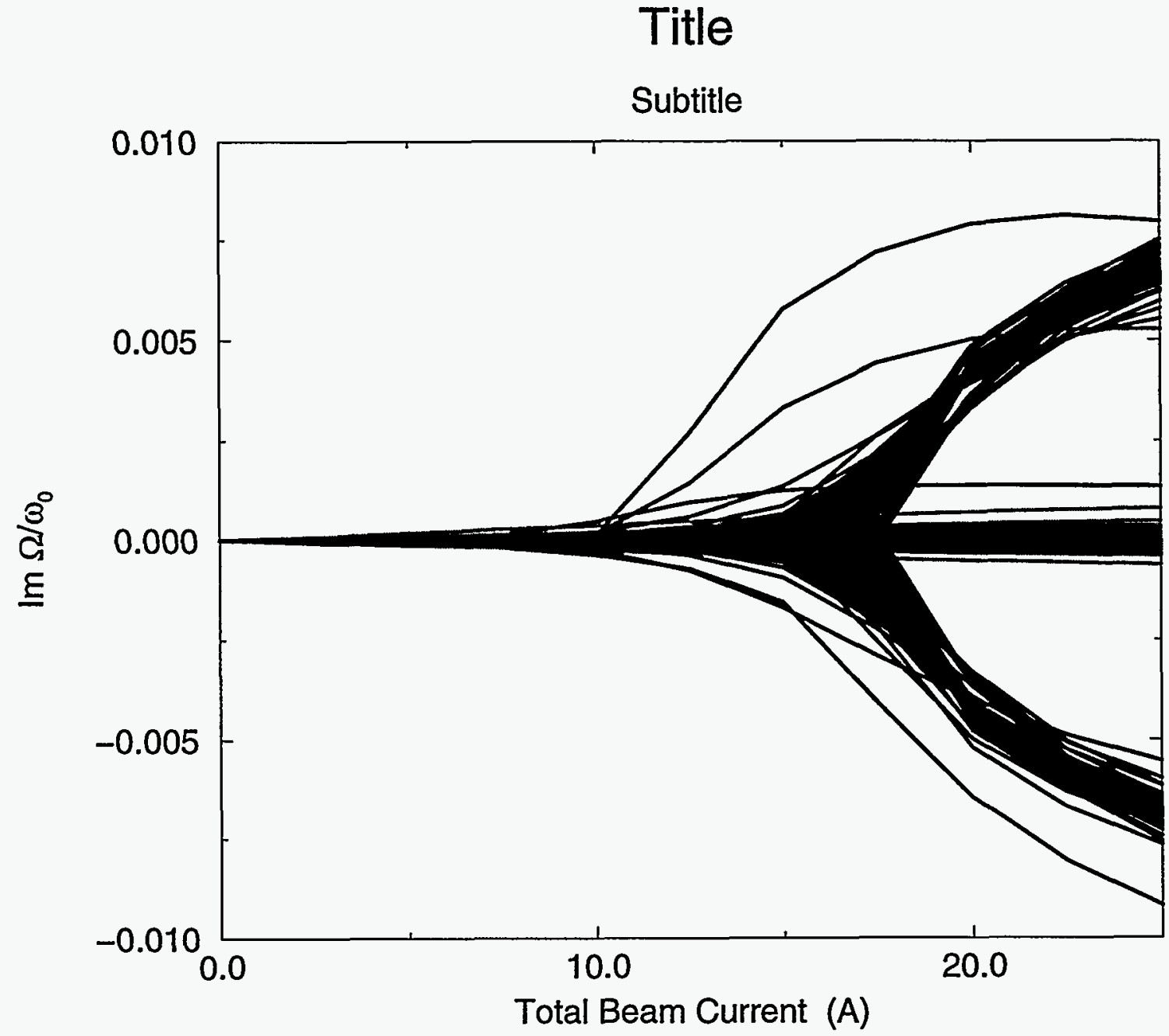

Figure 6: Output from "Modes vs. current, imag, one mode set."

ResistiveWall_II. There is one value, $R$, where the impedance is given by the formula

$$
-i \sqrt{2} \frac{R}{\sqrt{-i \omega / \omega_{0}}}
$$

Here $\omega_{0}$ is the revolution frequency. Thus, $R$ is the real or imaginary part of the impedance at $\omega_{0}$. Inductive_TI. There is only one value, $L$. The impedance is $-i L$, a constant.

CavityTail_TI. There are three values, the real and imaginary parts of $\omega_{R}$, followed by $R$, where the impedance is given by

$$
i \frac{R \omega_{0}}{\omega}\left(\frac{1}{\sqrt{1+\omega / \omega_{R}^{*}}}-\frac{1}{\sqrt{1-\omega / \omega_{R}}}\right) .
$$

Note that the imaginary part of $\omega_{R}$ should be negative, and the real part of $\omega_{R}$ should be positive. SingleBranch_TI. There are two parameters, $R$ and $\omega_{C}$, where the impedance is given by

$$
\frac{-i R}{\left(1-i \omega / \omega_{C}\right)^{3 / 2}}
$$




\subsection{Transverse Feedback}

Between the Transverse-Feedback and END keywords, there are a sequence of keywords which indicate what type of transverse impedance you are using. Each of these keywords will be followed by one or more values. One of these values will be the gain of the feedback. This value will be in units of Ohms, and should take into account the $\beta$-functions at the pickup and the kicker. Also, every feedback device will have a distance between the pickup and kicker associated with it. This will be the pickup to kicker distance multiplied by $2 \pi$ and divided by the ring circumference. Finally, all frequencies used are the actual frequency divided by the revolution frequency.

LowPass_TF. There are three parameters: the gain $A$, a half-bandwidth $\omega_{C}$, and the pickup to kicker distance.

The model used has a response of

$$
\frac{i A}{1-i \omega / \omega_{C}}
$$

Butterworth2.TF There are three parameters, the same as those for LowPass_TF, except the model has a response like

$$
\frac{i A e^{-i \sqrt{2} \omega / \omega_{C}}}{1-i \sqrt{2} \omega / \omega_{C}-\omega^{2} / \omega_{C}^{2}}
$$

This is a second-order Butterworth filter, with the time delay removed.

\subsection{Example}

Consider the following example: A ring has the following parameters:

\begin{tabular}{|c|c|}
\hline$f_{\mathrm{rf}}$ & $476 \mathrm{MHz}$ \\
$h_{\mathrm{rf}}$ & 3492 \\
$\eta_{C}$ & 0.00123208786107 \\
$L$ & $2199.318 \mathrm{~m}$ \\
$\nu_{y}$ & 36.64 \\
$\nu_{s}$ & .03362246 \\
$\sigma_{\ell}$ & $1 \mathrm{~cm}$ \\
$E$ & $3.109 \mathrm{GeV}$ \\
$\epsilon_{y}$ & $2.57 \mathrm{~nm}-\mathrm{rad}$ \\
$M$ & 1746 \\
$I$ & $2.254 \mathrm{~A}$ \\
\hline
\end{tabular}

The resistive wall impedance consists of two parts: a section with a total impedance of $902 \mathrm{k} \Omega / \mathrm{m}$ at the frequency $f_{\mathrm{rf}} / h_{\mathrm{rf}}$ with an average $\beta_{y}$ of $16.3061 \mathrm{~m}$, and a section with a total impedance of $560 \mathrm{k} \Omega / \mathrm{m}$ with an average $\beta_{y}$ of $22.1851 \mathrm{~m}$. There is an inductive impedance of $65.76 \mathrm{k} \Omega / \mathrm{m}$, which rolls off with a characteristic frequency near $24.1 \mathrm{GHz}$. Its average $\beta_{y}$ is $18.3647 \mathrm{~m}$. The high-frequency tails due to the cavities are modelled using equation (3), with $\omega_{R}=2.4-i 1.34722 \mathrm{GHz}$ and $R=15.7985 \mathrm{k} \Omega / \mathrm{m}$ per cavity. There are 8 cavities, with an average $\beta_{y}$ of $17.8720 \mathrm{~m}$. Those same cavities have the following higher-order modes:

\begin{tabular}{|c|c|c|}
\hline$f_{R}(\mathrm{GHz})$ & $Q$ & $R(\mathrm{k} \Omega / \mathrm{m})$ \\
\hline 0.792 & 115 & 34.916 \\
1.063 & 27 & 16.911 \\
1.133 & 54 & 0.162 \\
1.202 & 871 & 10.135 \\
1.327 & 611 & 58.328 \\
1.420 & 1138 & 160.689 \\
1.542 & 92 & 2.508 \\
1.595 & 145 & 7.777 \\
1.676 & 783 & 153.796 \\
1.723 & 446 & 0.177 \\
1.749 & 1317 & 2.414 \\
\hline
\end{tabular}


The following input file describes this ring:

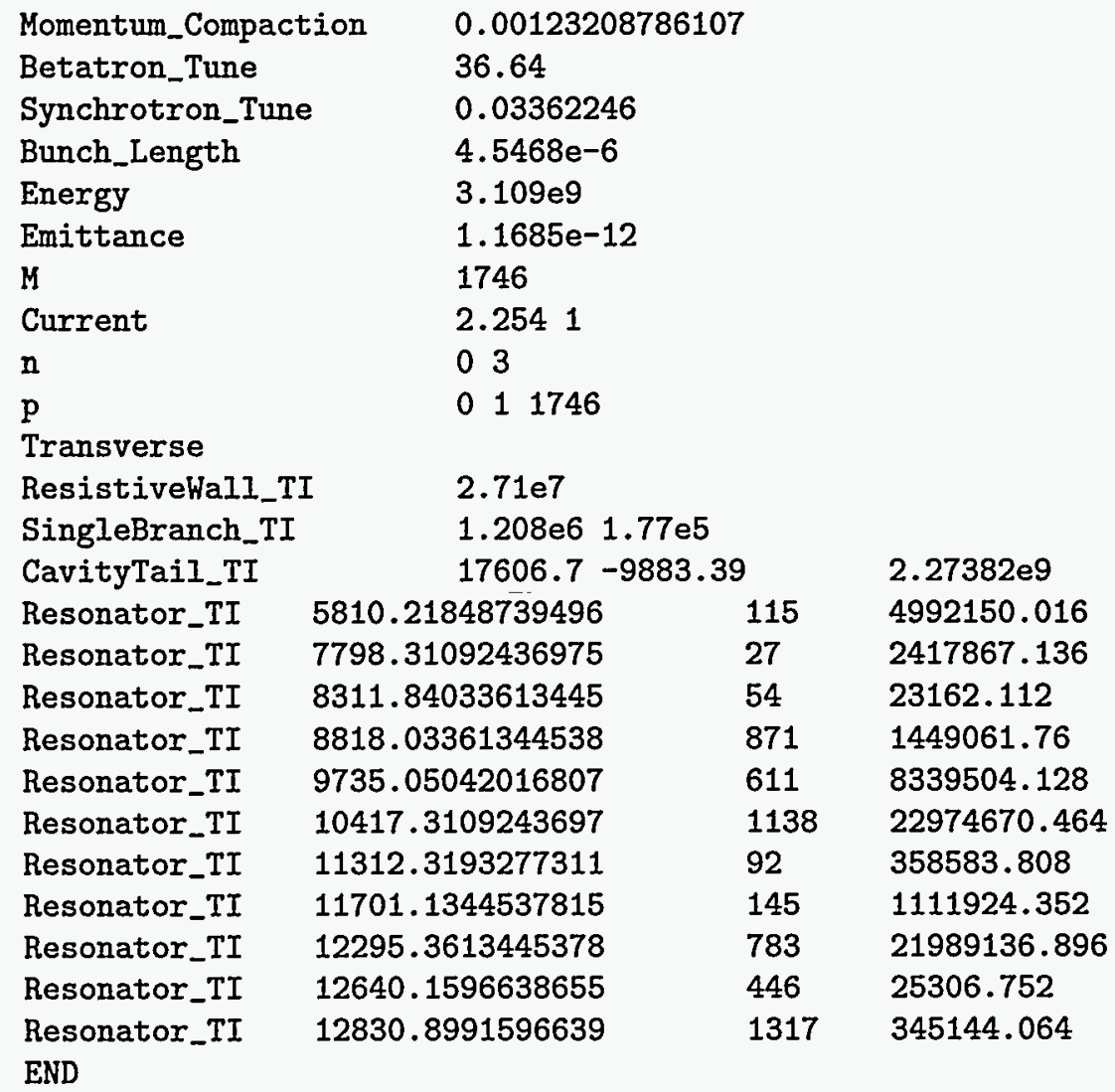

This input file will give a file with the $m=0, m=1, m=2, m=3, m=0$ radial, and $m=1$ radial modes at a single current of $2.254 \mathrm{~A}$, for all 1746 multibunch modes. If you want the modes for several currents, change the Current line to

Current 25100

which would find values for the modes for 100 evenly spaced currents up to $25 \mathrm{~A}$.

To add a $250 \mathrm{MHz}$ full-bandwidth damping feedback system, one would add the following lines

Transverse_Feedback

Butterworth2_TF 1e+07 9710.0428710787882

END

\section{Algorithm and Approximations}

This code uses the method described in [Ber96]. It computes the eigenvalues using equations (4.83) and (4.84), assuming that the elements computed in (4.85) are zero. It also assumes that (4.84) gives zero for $k \neq 0$. The dependence of $\sigma_{\tau}$ on $s$ is ignored, and $\Delta \psi_{\tau}$ is assumed to be zero. The bunches are assumed to be symmetric, and thus all the $I_{r}$ in equation (4.83) are taken to be the same. Also, the feedback contribution to the $Z$ 's described in equation (4.98) is included, with approximations similar to those for the impedance.

The code only computes the modes near $\Omega=\omega_{y}$. It evaluates all impedances and form factors at $\Omega=\omega_{y}$. Future updates will include code for finding the fixed point by evaluating the impedances and form factors at the computed $\Omega$.

\section{Getting the vlasov Code}

The vlasov package can be obtained by anonymous ftp to ftp.slac. stanford. edu, in the directory users/alhy. Get the file vlasov.tar.gz. The program is designed to be used in UNIX systems. To compile, the program 
requires gcc version 2.7 .2 or later, as well as libgt+ version 2.7 .1 or later. To view the plots, you need $x m g x$
version 3.01 , patch level 7 .

\section{References}

[Ber96] J. Scott Berg. Coherent Modes for Multiple Non-Rigid Bunches in a Storage Ring. PhD thesis, Stanford University, Stanford, CA, March 1996. SLAC report SLAC-R-478.

[BR95a] J. Scott Berg and Ronald D. Ruth. Transverse instabilities for multiple nonrigid bunches in a storage ring. Phys. Rev. E, 52(3):R2179-2182, September 1995.

[BR95b] J. Scott Berg and Ronald D. Ruth. Transverse multibunch modes for non-rigid bunches, including mode coupling. Technical Report SLAC-PUB-95-6965, SLAC, Stanford, CA, August 1995.

[Wie93] Helmut Wiedemann. Particle Accelerator Physics. Springer-Verlag, Berlin, 1993.

\section{DISCLAIMER}

This report was prepared as an account of work sponsored by an agency of the United States Government. Neither the United States Government nor any agency thereof, nor any of their employees, makes any warranty, express or implied, or assumes any legal liability or responsibility for the accuracy, completeness, or usefulness of any information, apparatus, product, or process disclosed, or represents that its use would not infringe privately owned rights. Reference herein to any specific commercial product, process, or service by trade name, trademark, ence heris to not necessarily constitute or imply its endorsement, recommanufacturer, or otherwise dhe United States Government or any agency thereof. The views mendation, or favoring by the Uned herein do not necessarily state or reflect those of the and opinions of authors expressed herein do not 
